\title{
Asociación entre geohelmintos y condiciones socioambientales en diferentes poblaciones humanas de Argentina
}

\author{
María Inés Gamboa, ${ }^{1}$ Leonora Eugenia Kozubsky, ${ }^{2}$ María Elena Costas, ${ }^{2}$ \\ Mariela Garraza, ${ }^{1}$ Marta Inés Cardozo, ${ }^{2}$ María Laura Susevich, ${ }^{1}$ \\ Paula Natalia Magistrello ${ }^{2}$ y Graciela Teresa Navone ${ }^{1}$
}

Forma de citar

Gamboa MI, Kozubsky LE, Costas ME, Garraza M, Cardozo MI, Susevich ML, et al. Asociación entre geohelmintos y condiciones socioambientales en diferentes poblaciones humanas de Argentina. Rev Panam Salud Publica. 2009;26(1):1-8.

RESUMEN Objetivos. Analizar la relación entre las especies de geohelmintos identificadas en poblaciones urbanas, suburbanas y rurales de las Provincias de Buenos Aires y Misiones, Argentina, y los aspectos socioambientales que favorecen la infestación por estos parásitos.

Métodos. Estudio transversal. Se analizaron 700 muestras fecales humanas provenientes de 319 familias residentes en una población urbana (LPU) y dos suburbanas (LPS, LPN) de la Provincia de Buenos Aires, y una población rural de la provincia de Misiones (MR), en Argentina. Se colectaron 35 muestras fecales de perros y 205 de tierra, y se completaron encuestas sobre las características socioambientales de las localidades estudiadas. Se utilizaron las técnicas de análisis parasitológicos de Ritchie, Carles Barthelemy, Fülleborn y Kato Katz.

Resultados. La frecuencia de parasitosis fue mayor en $M R(78,4 \%)$, seguida de las áreas suburbanas LPN (35,0\%) y LPS (25,8\%), y fue menor en la zona urbana LPU (5,7\%). Los ancilostomideos $(71,1 \%)$ y Strongyloides stercoralis $(22,2 \%)$ se detectaron solamente en MR y Ascaris lumbricoides, Hymenolepis nana y Trichuris trichiura fueron más frecuentes en LPN. Las muestras de heces de perros de Misiones presentaron una mayor frecuencia de parásitos (100\%) que las de las localidades de Buenos Aires, pero el suelo del sector urbano bonaerense resultó más contaminado. Las prácticas de alimentación y defecación poco higiénicas, el hacinamiento, el hábito de no usar calzado y el piso de tierra en las viviendas se asociaron significativamente con una mayor frecuencia de geohelmintos y pseudogeohelmintos intestinales $(\mathrm{P}<0,05)$. Conclusiones. El alto número de casos con infestación múltiple observado tanto en las poblaciones suburbanas como en la rural merece una atención especial y plantea la necesidad de profundizar estudios epidemiológicos que integren los diversos aspectos de este complejo problema a fin de implementar acciones y programas sanitarios eficaces.

Helmintos, condiciones sociales, Argentina.

1 Centro de Estudios Parasitológicos y de Vectores, Universidad Nacional de La Plata y Consejo Nacional de Investigaciones Científicas y Técnicas (CONICET), La Plata, Argentina. La correspondencia se debe dirigir a María I. Gamboa, Centro de Estudios Parasitológicos y de Vectores, Universidad Nacional de La Plata, Calle 2 No. 584, La Plata 1900, Buenos Aires, Argentina. Correo electrónico: inesgamboa@cepave.edu.ar

2 Cátedra de Parasitología, Facultad de Ciencias Exactas, Universidad Nacional de La Plata, La Plata, Buenos Aires, Argentina.
Las parasitosis provocadas por geohelmintos son endémicas en los países en desarrollo y constituyen un indicador de las condiciones sanitarias y ecológicas del entorno de sus hospederos (1). Los conglomerados urbanos con deficiente saneamiento ambiental y las características del ciclo de vida de estos parásitos intestinales determinan que las poblacio- nes más pobres sean las más vulnerables (1). Más de mil millones de personas en el mundo están infestadas por al menos una especie de geohelminto - entre las que se encuentran Ascaris lumbricoides, Trichuris trichiura, Strongyloides stercoralis y miembros de la familia Ancylostomidae- o pseudogeohelminto - Hymenolepis nana y especies de la familia Taenii- 
dae- (2, 3). En América Latina se estima que entre 10 y $20 \%$ de la población está infestada (4). Son frecuentes los casos de parasitismo múltiple (superinfestaciones o coinfestaciones, crónicas en algunos casos), con efectos insidiosos sobre el crecimiento, el estado nutricional y el desarrollo, especialmente cuando afectan a niños en edades preescolar y escolar (3, 5-8). En Argentina, la frecuencia de geohelmintosis es muy variable $(0,6-75,0 \%)$ y se distribuye de manera heterogénea a lo largo del país (9-13).

También las mascotas, principalmente los perros, desempeñan un papel importante en la transmisión de geohelmintos de importancia zoonótica. Estudios realizados en muestras de suelo han detectado parásitos capaces de infestar a humanos (14-22). Se ha demostrado que los huevos de Toxocara canis pueden infestar a un hospedero susceptible después de estar en el suelo un largo tiempo en condiciones extremas de temperatura y humedad $(15,17,19,23)$.

En el presente trabajo se analizó la relación entre las especies de geohelmintos identificadas en poblaciones urbanas, suburbanas y rurales de las provincias de Buenos Aires y Misiones, Argentina, y los aspectos socioambientales que podrían favorecer la infestación por estos parásitos.

\section{MATERIALES Y MÉTODOS}

Se realizó un estudio transversal basado en datos coproparasitológicos y las características socioambientales de tres barrios de la ciudad de La Plata, provincia de Buenos Aires, Argentina - una población urbana (LPU) y dos asentamientos suburbanos ubicados a $1 \mathrm{~km}$ al norte del casco urbano (LPN) y a $3 \mathrm{~km}$ al sur de la ciudad (LPS), respectivamente- y una población rural aborigen del municipio Aristóbulo del Valle, en la provincia de Misiones (MR).

La ciudad de La Plata tiene una población de 600000 habitantes, una temperatura promedio anual de $17^{\circ} \mathrm{C}$ y una humedad relativa de $78 \%$, con $1000 \mathrm{~mm}$ de precipitaciones anuales en promedio. El suelo predominante es de tipo argiudol, con abundante materia orgánica y textura franco-limosa (24). La cobertura del sistema de cloacas, alcantarillas y agua potable es de $100 \%$ en el área urbana y de 35 a 55\% en la zona periurbana; mientras que en los asentamientos precarios es prácticamente nula. La cantidad y ca- lidad de los servicios de salud en la población urbana (LPU) son insuficientes y se basan en los hospitales públicos y privados de la ciudad. En el asentamiento suburbano LPS hay un seguimiento periódico de la salud por parte de un médico pediatra que atiende en un comedor barrial, mientras que en LPN la población cuenta con una unidad sanitaria; ambos asentamientos se encuentran aproximadamente a $10 \mathrm{~km}$ de distancia del Río La Plata.

En el asentamiento rural (MR), el clima es cálido y húmedo con una temperatura media anual de $20^{\circ} \mathrm{C}$; las precipitaciones son abundantes, entre $1500 \mathrm{y}$ $2000 \mathrm{~mm}$ anuales. El suelo es profundo, de arena y arcilla, con una textura gruesa que dificulta la evaporación. La capa vegetal está formada por cuatro o cinco estratos verticales, con flora y fauna muy abundantes. En esta área rural hay asentamientos de varias comunidades aborígenes de la etnia mbyá guaraní, que tienen sus viviendas en claros abiertos en la selva. La comunidad aborigen analizada cuenta con un puesto de primeros auxilios a cargo de un agente sanitario aborigen, visitado muy esporádicamente por un profesional médico. Esta comunidad se encuentra a $12 \mathrm{~km}$ del hospital público de la ciudad Aristóbulo del Valle.

\section{Análisis socioambiental}

Se aplicó una encuesta a los jefes de hogar (319 en total) y se les entregaron frascos para obtener muestras seriadas de materia fecal de cada miembro de la familia menor de 60 años. Las encuestas indagaron sobre las condiciones socioambientales de las viviendas y las familias: materiales de construcción de la vivienda (paredes, techo y piso), servicios (agua de consumo, eliminación de los residuos, disposición de las excretas), hacinamiento (más de tres personas en una habitación), promiscuidad (más de una persona por cama simple), tipo de alimentación y uso de calzado. Se preguntó además por el nivel de educación y la actividad laboral de los padres (25).

\section{Análisis parasitológico}

Las muestras fecales humanas se tomaron diariamente durante cinco días y se sometieron a análisis coproparasitólógicos en busca de geohelmintos y pseudogeohelmintos. Se analizaron 35 muestras de perros (12 en LPS, 12 en LPN y 11 en
MR) recogidas en áreas peridomiciliarias. Con el fin de optimizar los resultados del análisis parasitológico, las muestras de heces (humanas y de perros) se analizaron mediante dos técnicas de sedimentación (Ritchie y Carles Barthelemy) y una de flotación (Fülleborn) (26-27). Las cargas parasitarias en muestras de heces humanas se estimaron mediante la técnica de Kato Katz (26). Para el análisis parasitológico del suelo se tomaron 205 muestras de tierra del entorno de las viviendas: 59 en MR, 28 en LPN, 18 en LPS y 100 en los espacios públicos de LPU. Las muestras se suspendieron en Tween $80 \mathrm{y}$ se analizaron mediante técnicas de concentración por flotación (13).

Para calcular el tamaño de la muestra en MR, LPS y LPN se utilizó la fórmula para estudios poblacionales del programa Epi Info 6.0, con un error esperado de $10 \%$, un intervalo de confianza de $99 \%$ y las prevalencias específicas de las localidades estudiadas (28). Como en LPU el número promedio de integrantes de las familias era menor que en las otras poblaciones, se tomó un número mayor de familias para que el número de personas a estudiar fuera homogéneo. Se analizó la asociación estadística entre las características ambientales (variables independientes) y las parasitosis detectadas (variables dependientes) mediante la prueba de la ji al cuadrado y sus intervalos de confianza de 95\% (IC95\%) mediante el programa Epi Info 6.0. El nivel de significación escogido fue de $P \leq 0,05$.

Todas las personas evaluadas dieron su consentimiento informado por escrito. Este trabajo recibió la aprobación de la Universidad Nacional de La Plata (Resol. HCS 1999-2001; 2002-2004) y la Comisión de Investigaciones Científicas de la Provincia de Buenos Aires.

\section{RESULTADOS}

Se investigaron 319 familias (42 en MR, 73 en LPN, 78 en LPS y 126 en LPU), lo que conformó una muestra total de 700 personas (194 en MR, 120 en LPN, 194 en LPS y 192 en LPU). No se observaron diferencias significativas entre las muestras estudiadas en las diferentes localidades en cuanto al sexo y la edad (cuadro 1).

De los dos asentamientos suburbanos de la ciudad de La Plata, LPS tenía mejores condiciones socioambientales que LPN y en ambos la situación era mejor que en MR (cuadro 1). Las poblaciones 
CUADRO 1. Características demográficas y socioambientales de las poblaciones estudiadas ${ }^{a}$

\begin{tabular}{|c|c|c|c|c|c|c|c|c|c|}
\hline \multirow[b]{2}{*}{ Característica } & \multicolumn{2}{|c|}{$\begin{array}{c}\text { MR } \\
(n=194)\end{array}$} & \multicolumn{2}{|c|}{$\begin{array}{c}\text { LPN } \\
(n=120)\end{array}$} & \multicolumn{2}{|c|}{$\begin{array}{c}\text { LPS } \\
(n=194)\end{array}$} & \multicolumn{2}{|c|}{$\begin{array}{c}\text { LPU } \\
(n=192)\end{array}$} & \multirow[b]{2}{*}{$P$} \\
\hline & No. & $\%$ & No. & $\%$ & No. & $\%$ & No. & $\%$ & \\
\hline Sexo & & & & & & & & & $>0,05$ \\
\hline Mujeres & 105 & 54,1 & 70 & 58,3 & 116 & 59,8 & 86 & 44,8 & \\
\hline Hombres & 89 & 45,9 & 50 & 41,7 & 78 & 40,2 & 106 & 55,2 & \\
\hline Edad (años) & & & & & & & & & $>0,05$ \\
\hline $0-4$ & 61 & 31,4 & 52 & 43,3 & 94 & 48,4 & 68 & 35,4 & \\
\hline $5-9$ & 70 & 36,0 & 39 & 32,5 & 56 & 28,9 & 81 & 42,2 & \\
\hline $10-14$ & 29 & 15,1 & 22 & 18,3 & 12 & 6,2 & 30 & 15,6 & \\
\hline$\geq 15$ & 34 & 17,5 & 7 & 5,9 & 32 & 16,5 & 13 & 6,8 & \\
\hline Materiales de la vivienda ${ }^{b}$ & & & & & & & & & $<0,01$ \\
\hline Precarios (chapa y madera) & 194 & 100,0 & 98 & 81,7 & 116 & 59,8 & 49 & 25,5 & \\
\hline Ladrillos & 0 & 0,0 & 22 & 18,3 & 78 & 40,2 & 143 & 74,5 & \\
\hline Piso de la vivienda ${ }^{b}$ & & & & & & & & & $<0,01$ \\
\hline Tierra & 194 & 100,0 & 44 & 36,7 & 20 & 10,3 & 8 & 4,2 & \\
\hline Cemento u otros & 0 & 0,0 & 76 & 63,3 & 174 & 89,7 & 184 & 95,8 & \\
\hline Hacinamiento ${ }^{b}$ & & & & & & & & & $<0,01$ \\
\hline Sí & 194 & 100,0 & 100 & 83,3 & 114 & 58,8 & 63 & 32,8 & \\
\hline No & 0 & 0,0 & 20 & 16,7 & 80 & 41,2 & 129 & 67,2 & \\
\hline Promiscuidad ${ }^{b}$ & & & & & & & & & $<0,01$ \\
\hline Sí & 194 & 100,0 & 84 & 70,0 & 114 & 58,8 & 44 & 22,9 & \\
\hline No & 0 & 0,0 & 36 & 30,0 & 80 & 41,2 & 148 & 77,1 & \\
\hline Eliminación de excretas ${ }^{b}$ & & & & & & & & & $<0,01$ \\
\hline Cielo abierto o letrina & 194 & 100,0 & 91 & 75,8 & 70 & 36,3 & 16 & 8,3 & \\
\hline Pozo ciego o cloacas & 0 & 0,0 & 29 & 24,2 & 124 & 63,7 & 176 & 91,7 & \\
\hline Uso de calzado b & & & & & & & & & $<0,01$ \\
\hline No & 194 & 100,0 & 0 & 0,0 & 0 & 0,0 & 0 & 0,0 & \\
\hline Sí & 0 & 0,0 & 120 & 100,0 & 194 & 100 & 192 & 100,0 & \\
\hline Actividad laboral $^{b}$ & & & & & & & & & $<0,01$ \\
\hline Desempleado, cirujeo ${ }^{C}$ o inestable & 194 & 100,0 & 80 & 66,7 & 122 & 62,9 & 29 & 15,1 & \\
\hline Trabajo estable o independiente & 0 & 0,0 & 40 & 33,3 & 72 & 37,1 & 163 & 84,9 & \\
\hline Nivel educacional ${ }^{b}$ & & & & & & & & & $<0,01$ \\
\hline Analfabeto & 116 & 60,0 & 20 & 16,7 & 0 & 0,0 & 0 & 0,0 & \\
\hline Primario & 78 & 40,0 & 86 & 71,7 & 150 & 77,3 & 85 & 44,3 & \\
\hline Secundario o mayor & 0 & 0,0 & 14 & 11,6 & 44 & 22,7 & 107 & 55,7 & \\
\hline Agua $^{d}$ & & & & & & & & & $<0,01$ \\
\hline Acarreo & 194 & 100,0 & 16 & 13,3 & 1 & 0,5 & 0 & 0,0 & \\
\hline Bomba & 0 & 0,0 & 0 & 0,0 & 2 & 1,0 & 5 & 2,6 & \\
\hline Red de agua corriente & 0 & 0,0 & 104 & 86,7 & 191 & 98,5 & 187 & 97,4 & \\
\hline Eliminación de residuos ${ }^{b}$ & & & & & & & & & $<0,01$ \\
\hline Cielo abierto, quema o enterramiento & 194 & 100,0 & 120 & 100,0 & 44 & 22,7 & 2 & 1,0 & \\
\hline Recolección municipal & 0 & 0,0 & 9 & 0,0 & 150 & 77,3 & 190 & 99,0 & \\
\hline
\end{tabular}

suburbanas (LPS y LPN) carecían por lo general de conexión con la red cloacal y eliminaban las excretas mediante pozos ciegos, letrinas o a cielo abierto. La población de LPU, por su parte, era totalmente urbana y se servía de los servicios y la infraestructura de la ciudad (salvo muy pocos casos de viviendas precarias que no tenían conexión con la red cloacal).

En LPS, 62,9\% de los padres no tenía trabajo estable y aunque $77,3 \%$ realizó estudios primarios, $27,3 \%$ de ellos no los completó; en LPN, 66,7\% de los padres estaba desocupado o se dedicaba al cirujeo (actividad que consiste en recorrer las ciudades con carros de tracción humana o animal para recoger, reciclar y vender residuos urbanos); $84,9 \%$ de la población en LPU contaba con una actividad laboral estable.

En MR, la única localidad completamente rural, todas las viviendas eran precarias, construidas de madera $y$ chapa y tenían piso de tierra. Todas las familias ocupaban viviendas pequeñas, con muestras claras de hacinamiento y promiscuidad. Estas familias defecaban a cielo abierto, en los alrededores de las viviendas. El agua para consumo se tomaba de un arroyo (Cuña Pirú) o de aljibes y se conservaba en recipientes a la intemperie durante varios días hasta su consumo. La población estaba compuesta en su mayoría por cazadores y recolectores, aunque temporalmente trabajaban en la cosecha de yerba mate y té (29). La mayoría de las personas de esta población no utilizaba calzado, aunque algunos lo usaban cuando iban a la ciudad. Los residuos sólidos los enterraban o quemaban.

De las 700 muestras fecales humanas analizadas, en las de MR se encontraron parásitos (A. lumbricoides, H. nana, T. trichiura, S. stercoralis y ancilostomideos) con mayor frecuencia $(78,4 \%)$ que en las del resto de las poblaciones estudiadas (LPN: 35,0\%; LPS: 25,8\%; y LPU: 5,7\%). 
Del total de muestras infestadas de cada población, 74,4\% (MR), 33,3\% (LPN), 30\% (LPS) y $9 \%$ (LPU) presentaron más de una especie de helminto, con diferencias en cuanto a la composición y distribución de las especies parasitarias. Si bien las especies coinfestantes en MR eran $H$. nana, A. lumbricoides, ancilostomideos y $S$. stercoralis; en LPN y LPS eran H. nana, $A$. lumbricoides y T. trichiura; y en LPU eran $H$. nana y A. lumbricoides. En general, la especie detectada con mayor frecuencia en las muestras de LPN, LPS y LPU fue A. lumbricoides, seguida por $H$. nana y $T$. trichiura, mientras que en MR fueron los ancilostomideos seguidos por S. stercoralis e H. nana (cuadro 2).

Se halló asociación estadísticamente significativa entre la presencia de A. lumbricoides, H. nana y T. trichiura en LPS y LPN $(P<0,01)$; entre $A$. lumbricoides, H. nana, $\mathrm{y}$ ancilostomideos $(P<0,05)$, así como entre $T$. trichiura y $S$. stercoralis en MR $(P<0,01)$. Al comparar la presencia de parasitosis por especie en las cuatro poblaciones estudiadas, se encontraron diferencias significativas en todas las especies, excepto A. lumbricoides. Hubo casos esporádicos de infestación por ancilostomideos y S. stercoralis en LPN, LPS y LPU. T. trichiura fue significativamente más frecuente en LPN que en MR $(P<0,01)$. Por su parte, $H$. nana fue menos frecuente en LPS que en el resto de las localidades estudiadas $(P<0,01)$. En LPU se observaron frecuencias significativamente inferiores $(P<0,01)$ que en el resto de las localidades, independientemente de la especie (cuadro 2).

En cuanto a la intensidad de la infestación, fue leve en LPS y LPU, mientras que en LPN y MR hubo casos de infestaciones moderadas e intensas (cuadro 2).

En las heces de perros, la frecuencia de los parásitos estudiados fue de $100 \%$ en MR, 33,3\% en LPS y $41,7 \%$ en LPN (no se investigó en LPU), con predominio de especies de transmisión zoonótica (cuadro 3). Las especies más frecuentes fueron los ancilostomideos, especialmente en MR, donde se observó una frecuencia significativamente mayor que en las localidades suburbanas $(P<0,01)$. La frecuencia de Trichuris vulpis y Toxocara canis fueron también superiores en MR, pero sin diferencias significativas con respecto a las otras dos poblaciones $(P>0,05)$. No se hallaron diferencias significativas entre las frecuencias de parásitos observadas en las poblaciones humanas y caninas.

Se encontraron geohelmintos y pseudogeohelmintos en $47,5 \%$ de las muestras de suelo de MR, 53,6\% de las de LPN, 66,7\% de las de LPS y 91,0\% de las de LPU (cuadro 3).

El hacinamiento y el sistema precario de eliminación de excretas se asociaron significativamente con las parasitosis en todas las poblaciones estudiadas. Por otra parte, la alimentación con sobrantes, el piso de tierra en las viviendas y la falta de calzado, particularidades de algunas de las poblaciones analizadas, también mostraron una asociación positiva (cuadro 4).

\section{DISCUSIÓN}

Según los resultados de este estudio, los geohelmintos y pseudogeohelmintos fueron más frecuentes en la población rural de la Provincia de Misiones (MR) que en las localidades suburbanas de la Provincia de Buenos Aires (78,4\% frente a $35,0 \%$ en LPN y $25,8 \%$ en LPS) y la composición específica fue diferente. En este sentido, las frecuencias observadas en la comunidad asentada en MR se encuentran entre las más altas descritas para este tipo de población (30-32).

El valor de infestación hallado en uno de los sectores suburbanos de la provincia de Buenos Aires (LPN) es cercano al informado para la ciudad de Santa Fe $(34 \%)$, ubicada en la provincia de Santa Fe (10). Sin embargo, estudios realizados en Bahía Blanca, al sur de la provincia de Buenos Aires, con un clima más frío que el predominante en La Plata, mostraron frecuencias inferiores (entre 1,0 y $24,5 \%$ ) (9). Asimismo, Soriano y colaboradores (33) no encontraron geohelmintos cuando analizaron la población infantil y el suelo de la provincia de Neuquén, en el noreste de la Patagonia argentina, con un clima frío y una fitogeografía diferente a la de Buenos Aires. El clima seco, ya sea templado o frío, parece no favorecer el desarrollo de los geohelmintos (23)

CUADRO 2. Frecuencias absoluta y relativa (\%) de geohelmintosis y pseudogeohelmintosis humanas en las cuatro poblaciones estudiadas ${ }^{a}$

\begin{tabular}{|c|c|c|c|c|c|c|c|c|c|c|c|c|c|c|c|c|c|c|c|c|}
\hline \multirow[b]{3}{*}{ Especie } & \multirow[b]{3}{*}{ No. } & \multicolumn{4}{|c|}{$\operatorname{MR}(n=194)^{\mathrm{b}}$} & \multicolumn{5}{|c|}{$\operatorname{LPN}(n=120)^{c}$} & \multicolumn{5}{|c|}{$\operatorname{LPS}(n=194)^{c}$} & \multicolumn{5}{|c|}{ LPU $(n=192)$} \\
\hline & & & $\begin{array}{l}\text { Inten } \\
\text { infe }\end{array}$ & $\begin{array}{l}\text { sidad c } \\
\text { tación, }\end{array}$ & & \multirow[b]{2}{*}{ No. } & \multirow[b]{2}{*}{$\%$} & \multicolumn{3}{|c|}{$\begin{array}{l}\text { Intensidad de la } \\
\text { infestación, d \% }\end{array}$} & \multirow[b]{2}{*}{ No. } & \multirow[b]{2}{*}{$\%$} & \multicolumn{3}{|c|}{$\begin{array}{l}\text { Intensidad de la } \\
\text { infestación, }{ }^{\text {d \% }}\end{array}$} & \multirow[b]{2}{*}{ No. } & \multirow[b]{2}{*}{$\%$} & \multicolumn{3}{|c|}{$\begin{array}{l}\text { Intensidad de la } \\
\text { infestación, }{ }^{\circ} \%\end{array}$} \\
\hline & & $\%$ & L & M & I & & & L & M & I & & & L & M & 1 & & & L & $\mathrm{M}$ & I \\
\hline \multicolumn{21}{|l|}{ Ascaris } \\
\hline & 25 & 12,9 & 76,0 & 16,0 & 8,0 & 16 & 21,6 & 81,2 & 12,5 & 6,3 & 36 & 18,6 & 100,0 & 0,0 & 0,0 & 8 & 4,2 & 100,0 & 0,0 & 0,0 \\
\hline trichiura $^{\mathrm{e}}$ & 6 & 3,1 & 100,0 & 0,0 & 0,0 & 12 & 10,0 & 100,0 & 0,0 & 0,0 & 11 & 5,7 & 100,0 & 0,0 & 0,0 & 0 & 0,0 & 0,0 & 0,0 & 0,0 \\
\hline $\begin{array}{r}\text { stercoralis } \\
\text { Hymenolepis }\end{array}$ & 43 & 22,2 & 93,0 & 0,0 & 7,0 & 0 & 0,0 & 0,0 & 0,0 & 0,0 & 2 & 1,0 & 0,0 & 0,0 & 0,0 & 0 & 0,0 & 0,0 & 0,0 & 0,0 \\
\hline nana $^{f}$ & 38 & 19,6 & 0,0 & 0,0 & 0,0 & 21 & 17,5 & 0,0 & 0,0 & 0,0 & 18 & 9,3 & 0,0 & 0,0 & 0,0 & 4 & 2,1 & 0,0 & 0,0 & 0,0 \\
\hline Total $^{c}$ & 152 & 78,4 & 0,0 & 0,0 & 0,0 & 42 & 35,0 & 0,0 & 0,0 & 0,0 & 50 & 25,8 & 0,0 & 0,0 & 0,0 & 11 & 5,7 & 0,0 & 0,0 & 0,0 \\
\hline
\end{tabular}

a MR: comunidad rural de Aristóbulo del Valle, Misiones; LPN: asentamiento suburbano al norte de La Plata, Buenos Aires; LPS: asentamiento suburbano al sur de La Plata; LPU: comunidad urbana de La Plata.

b Según la prueba de la ji al cuadrado se encontró asociación entre la presencia de Ascaris lumbricoides, Hymenolepis nana y ancilostomideos $(P<0,01)$.

${ }^{c}$ Según la prueba de la ji al cuadrado se encontró asociación entre la presencia de Ascaris lumbricoides, Hymenolepis nana y Trichuris trichiura $(P<0,01)$

d Intensidad de la infestación: L: leve; M: moderada; I: intensa.

e Según la prueba de la ji al cuadrado se encontró diferencia significativa entre los niveles de infestación en MR y LPN $(P<0,01)$

f Según la prueba de la ji al cuadrado se encontró diferencia significativa entre los niveles de infestación en las cuatro poblaciones $(P<0,01)$. 
CUADRO 3. Frecuencias absoluta y relativa (\%) de geohelmintos y pseudogeohelmintos en las muestras de heces de perros y de tierra en las poblaciones estudiadas ${ }^{\mathrm{a}}$

\begin{tabular}{|c|c|c|c|c|c|c|c|c|c|}
\hline \multirow[b]{2}{*}{ Especie } & \multicolumn{2}{|c|}{ MR } & \multicolumn{2}{|c|}{ LPN } & \multicolumn{2}{|c|}{ LPS } & \multicolumn{2}{|c|}{ LPU } & \multirow[b]{2}{*}{$P^{\mathrm{b}}$} \\
\hline & No. & $\%$ & No. & $\%$ & No. & $\%$ & No. & $\%$ & \\
\hline \multicolumn{10}{|l|}{ En perros } \\
\hline Toxocara canis & 3 & 27,3 & 2 & 16,7 & 1 & 8,3 & NA & NA & $>0,05$ \\
\hline Trichuris vulpis & 1 & 9,1 & 1 & 8,3 & 0 & 0 & NA & NA & $>0,05$ \\
\hline Ascaris spp. & 1 & 9,1 & 2 & 16,7 & 0 & 0 & NA & NA & $>0,05$ \\
\hline Larvas de nematodos & 2 & 18,2 & 0 & 0 & 1 & 8,3 & NA & NA & $>0,05$ \\
\hline Toxocara spp. ${ }^{\mathrm{e}}$ & 11 & 18,6 & 9 & 32,1 & 1 & 5,6 & 13 & 13,0 & $<0,05$ \\
\hline Ascaris lumbricoides & 10 & 16,9 & 8 & 28,6 & 2 & 11,1 & 21 & 21,0 & $>0,05$ \\
\hline Trichuris spp. & 6 & 10,2 & 0 & 0 & 1 & 5,6 & 4 & 4,0 & $>0,05$ \\
\hline Ancilostomideos ${ }^{\dagger}$ & 9 & 15,3 & 3 & 10,7 & 0 & 0 & 2 & 2,0 & $<0,01$ \\
\hline Taenia spp. & 0 & 0 & 2 & 7,1 & 0 & 0 & 2 & 2,0 & $>0,05$ \\
\hline Hymenolepis nana & 1 & 1,7 & 0 & 0 & 0 & 0 & 1 & 1,0 & $>0,05$ \\
\hline Larvas de nematodos libres & 41 & 69,5 & 15 & 53,6 & 12 & 66,7 & 46 & 46,0 & $>0,05$ \\
\hline
\end{tabular}

${ }^{a}$ MR: comunidad rural de Aristóbulo del Valle, Misiones; LPN: asentamiento suburbano al norte de La Plata, Buenos Aires; LPS: asentamiento suburbano al sur de La Plata; LPU: comunidad urbana de La Plata.

${ }^{\text {b }}$ Según la prueba de la ji al cuadrado. Nivel de significación $P<0,05$.

${ }^{c}$ Diferencia significativa entre las frecuencias de infestación de las 4 poblaciones $(P<0,01)$.

¿NA: no se analizó.

e Diferencia significativa entre las frecuencias de infestación en LPN y LPS $(P<0,05)$.

'Diferencia significativa entre las frecuencias de infestación en MR y LPU $(P<0,01)$.

CUADRO 4. Asociación entre las variables socioambientales y la frecuencia relativa (\%) de geohelmintos y pseudogeohelmintos en las localidades estudidas $^{\mathrm{a}}$

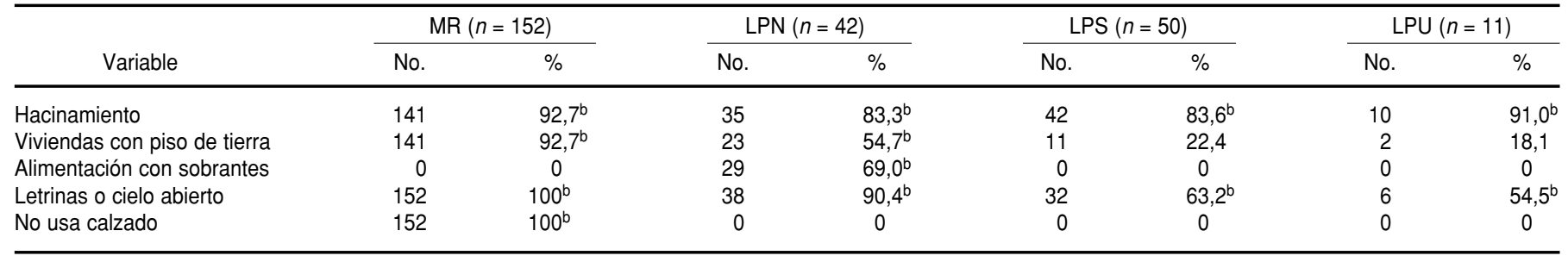

a MR: comunidad rural de Aristóbulo del Valle, Misiones; LPN: asentamiento suburbano al norte de La Plata, Buenos Aires; LPS: asentamiento suburbano al sur de La Plata; LPU: comunidad urbana de La Plata.

${ }^{b}$ Diferencia significativa entre infestados y no infestados en esa localidad, según la prueba de la ji al cuadrado $(P<0,05)$.

Cuando se compararon los porcentajes de parasitismo en las tres poblaciones estudiadas en la provincia de Buenos Aires, se observó una frecuencia inferior en la población urbana LPU $(5,7 \%)$ que en las suburbanas LPS $(25,8 \%)$ y LPN $(35,0 \%)$. Esto puede deberse a los factores sociales relacionados con la atención de la salud, el estado de las viviendas, las condiciones sanitarias y los hábitos de conducta de la población, que mostraron diferencias significativas entre estas tres poblaciones. Por ejemplo, en los asentamientos suburbanos se observaron conexiones a la red de agua potable elaboradas de manera clandestina por los pobladores, compuestas por cañerías de plástico, en su mayoría apoyadas en la tierra o conectadas a una llave de agua fuera o dentro de las viviendas. Las diversas formas de alimentación también podrían influir en las diferencias observadas, ya que en LPN se consumen frutas y verduras provenientes de la recolección de sobrantes del mercado local, algo que no se observó en las otras áreas estudiadas.

La población rural de Misiones (MR) presentó un marcado predominio de ancilostomideos y $S$. stercoralis sobre las restantes especies, con altas tasas de infestación múltiple $(74,4 \%)$. Esta situación fue similar a la observada en la comunidad wichí de Orán, provincia de Salta, en el extremo norte de Argentina —que tiene un clima subtropical cálido-, donde la frecuencia de infestación múltiple es alta $(70 \%)$, con predominio tam- bién de ancilostomideos y $S$. stercoralis (12). En MR, las frecuencias de A. lumbricoides y $T$. trichiura fueron más bajas que las encontradas en otras poblaciones de las provincias de Buenos Aires, Santa Fe y Corrientes $(10,12,34)$. Las diferencias observadas en cuanto a la distribución de las especies de parásitos pueden deberse a las condiciones de humedad y temperatura ambiental, así como al tipo de sustrato en el cual se desarrollan y transmiten los huevos y las larvas. Si se toma en cuenta que los huevos de los geohelmintos necesitan pasar un tiempo en el suelo para tener la capacidad de infestar a humanos, su desarrollo y viabilidad dependerán de las condiciones que este sustrato les brinde. Las larvas de ancilostomideos y S. stercoralis, por ejemplo, tie- 
nen una vida corta en el suelo, ya que generalmente no sobreviven más de un mes (35). Está demostrado que la humedad y la temperatura elevadas, unidas al hábito de defecar a cielo abierto y andar descalzos, favorecen la transmisión y dispersión de ancilostomideos y $S$. stercoralis $(13,35)$. Estas condiciones predominaban en MR, donde las frecuencias de ancilostomideos y S. stercoralis fueron más elevadas, pero no favorecían el desarrollo de A. lumbricoides y T. trichiura, cuyas frecuencias fueron más bajas que las observadas en otras provincias argentinas $(10,12,30)$.

Con respecto al control de estas parasitosis, Sorensen y colaboradores (35) consideran que el establecimiento de las condiciones sanitarias adecuadas permite reducir rápidamente su incidencia. Sin embargo, según estudios realizados sobre los hábitos de conducta en poblaciones mbya guaraní, los elevados niveles de parasitismo solo se podrán controlar mediante quimioterapia asistida periódica (13). Esta situación, si bien no resuelve el problema de base, permitiría controlar la infestación existente y retardar la reinfestación, que resulta inevitable dadas las condiciones ambientales y los hábitos de estas poblaciones. La frecuencia de infestación por S. stercoralis y ancilostomideos en las poblaciones estudiadas de Buenos Aires fue muy baja (menor de 2,0\%), lo cual confirma la influencia del ambiente en la distribución de estos parásitos.

Por otra parte, los huevos de Hymenolepis spp., Ascaris spp. y Trichuris spp. sobreviven mucho más tiempo en el suelo y su detección en humanos está generalmente asociada con las condiciones de hacinamiento (35). En los asentamientos suburbanos bonaerenses la frecuencia de A. lumbricoides en heces humanas varió entre $18,6 \%$ y $21,6 \%$, mayor que la observada en el sur de la provincia de Buenos Aires (8,8\%) (9) y en Salta (2,1\%) (12), pero menor que la hallada en Santa Fe $(31,4 \%)$ (10). En lo que respecta a $H$. nana, los valores hallados en esos asentamientos (entre 9,3\% y 17,5\%) fueron semejantes a los informados para Buenos Aires (24,5\%) (9) y mayores que los observados en las provincias de Santa Fe (4,3\%-18,2\%) (10) y Salta $(11,6 \%)(12)$. Sin embargo, lo observado en el área urbana estudiada (LPU) se aparta de esta tendencia, ya que las frecuencias observadas fueron inferiores para todas las especies estudiadas. Los resultados obtenidos en el presente trabajo y los obtenidos en otras investigaciones de Argentina demuestran que las condiciones socioambientales de las poblaciones analizadas determinan la presencia y frecuencia de estas especies de geohelmintos y pseudogeohelmintos.

La asociación hallada entre la frecuencia de A. lumbricoides, H. nana y T. trichiura, y de ancilostomideos con $H$. nana y A. lumbricoides, podría indicar que la coexistencia de estas especies no es casual. En este sentido, Ashford y colaboradores consideran que la asociación estadística entre pares de especies podría explicarse por la heterogeneidad ambiental y la coincidencia epidemiológica, sin que esto quiera decir que la infestación por unas especies influye en la infestación por otras (36). En la población urbana (LPU) no se encontró asociación estadística en la presencia de las diferentes especies, probablemente debido a la baja frecuencia detectada en esta población.

Las cargas parasitarias encontradas reflejaron el patrón de distribución agregada que generalmente siguen estos parásitos: pocos casos con infestación intensa y muchos con infestación leve.

La frecuencia de especies parasitarias en heces de perros fue mayor en el área rural de Misiones (100\%) que en los barrios suburbanos de la ciudad de La Plata (LPN: 41,7\% y LPS: 33,3\%). Probablemente las características climáticas predominantes en el noreste argentino y la falta de control sanitario de los perros puedan explicar estos resultados. En estudios previos se detectaron elevadas frecuencias de ancilostomideos en las heces de perros en aceras y espacios públicos de las localidades de Corrientes y $\mathrm{La}$ Plata $(22,37)$. Estos resultados muestran que tanto en la ciudad como en las áreas suburbanas y rurales hay un alto nivel de contaminación parasitaria por heces de perros (15). La detección de huevos de A. lumbricoides en las heces de perros de MR y LPN se podría explicar por el hábito de coprofagia de heces humanas (13). Este comportamiento se ve favorecido por la práctica de los pobladores de defecar a cielo abierto (1) y confirma el papel que desempeñan los perros en la propagación de los geohelmintos (19). Asimismo, algunas especies halladas en las heces caninas - como Toxocara canis y Ancylostoma caninum - pueden causar enfermedades al hombre, como larva migrans visceral, ocular, neurológica o cutánea, lo que agrava el riesgo en que se encuentran estas poblaciones. La ausencia de una asociación estadística entre la frecuencia de geohelmintos en humanos y perros podría deberse al bajo número de muestras caninas analizadas. Se requieren estudios a mayor escala para evaluar la importancia epidemiológica de estas infestaciones.

El análisis del suelo y la identificación de formas parasitarias es imprescindible para comprender la frecuencia de estas infestaciones en las poblaciones humanas $(13,15)$. La mayoría de los géneros de parásitos observados en las poblaciones analizadas estuvieron presentes en las muestras de suelo analizadas. La mayor frecuencia de estos parásitos en las muestras de tierra se encontró en las provenientes de la zona urbana (LPU), seguida por las de los barrios suburbanos (LPN y LPS) y las de la zona rural estudiada (MR). Este resultado tiene su explicación en el origen de las muestras, ya que las del casco urbano provenían de los parques públicos, mientras que las de los barrios suburbanos se tomaron en los alrededores de los domicilios. De este modo, los parques de la ciudad, sin reglamentación vigente para el control de la defecación de las mascotas, constituyen un riesgo mayor en la transmisión de la infestación parasitaria (15).

En el presente trabajo se confirmó la asociación existente entre la frecuencia de infestación con geohelmintos y pseudogeohelmintos, por una parte, y el tipo de alimentación, el piso de tierra de las viviendas, el hacinamiento, el uso de letrinas o la defecación a cielo abierto, y la ausencia de calzado, por otra $(10,11,13,38)$. El clima cálido y húmedo y el suelo arcilloso y poco permeable predominantes en Misiones ofrecen un ambiente adecuado para la supervivencia de los huevos y las larvas de estos parásitos y favorecen su propagación a grandes distancias. Los hábitos de defecación de la población estudiada (MR), la contaminación de las viviendas, las actividades que desarrollan en los alrededores del domicilio y el andar descalzo favorecen la infestación directa con algunos parásitos (ancilostomideos y S. stercoralis) al ingresar las larvas por la piel delgada que se encuentra entre los dedos. Estos factores, sumados al hacinamiento, convierten a la población rural de Misiones en la más vulnerable de las estudiadas. Por otra parte, la alimentación con sobrantes del mercado de verduras local en LPN también se asoció con la frecuencia de estas parasitosis. Esta situación podría agravarse ante la falta de 
higiene en los contenedores de alimentos del mercado y el lavado insuficiente de los sobrantes manipulados por las familias que los consumen.

Los resultados de este trabajo y el conocimiento acumulado a partir de otros estudios realizados en diferentes partes de Argentina permiten concluir que la distribución geográfica de los ancilostomideos y $S$. stercoralis depende en gran medida de las condiciones climáticas, el tipo de suelo y el comportamiento de las poblaciones susceptibles, y su frecuencia disminuye a medida que se avanza hacia el sur del país. Por su parte, la frecuencia de A. lumbricoides, T. trichiura e H. nana depende de factores socioambientales, como el hacina- miento, la alimentación, las condiciones de la vivienda, la higiene y el método de disposición de excretas empleado.

El alto número de casos con infestación múltiple observado tanto en las poblaciones suburbanas como en la rural merece una atención especial y plantea la necesidad de profundizar estudios epidemiológicos que integren los diversos aspectos de este complejo problema a fin de implementar acciones y programas sanitarios eficaces.

Se requieren mejores controles sanitarios y la promoción de prácticas adecuadas para la disposición de las excretas humanas y animales. Paralelamente se deben desarrollar planes de educación sanitaria y fomentar el concepto de responsabilidad individual y colectiva en la sanidad ambiental.

Agradecimientos. Los autores agradecen a las familias de las cuatro poblaciones estudiadas, al personal de los dos comedores de la Obra del Padre Cajade en La Plata y a Carlos Bertolotti, médico pediatra, por su colaboración desinteresada. Esta investigación recibió financiamiento parcial de la Comisión de Investigaciones Científicas de la Provincia de Buenos Aires, el Consejo Nacional de Investigaciones Científicas y Técnicas (CONICET) y la Universidad Nacional de La Plata.

\section{REFERENCIAS}

1. Traub RJ, Robertson ID, Irwin P, Mencke N, Thompson RCA. The prevalence, intensities, and risk factors associated with geohelminth infection in tea-growing communities of Assam, India. Trop Med Int Health. 2004;9: 688-701.

2. World Health Organization. Informal consultation on intestinal protozoal infections. México, D.F.: Pan American Health Organization; 1992.

3. World Health Organization. Deworming for health and development. Report of the Third Global Meeting of the Partners for Parasite Control. Geneva: WHO; 2005.

4. De Silva NR, Brooker S, Hotez PJ, Montresor A, Engels D, Savioli L. Soil-transmitted helminth infections: updating the global picture. Trends Parasitol. 2003;9:47-51.

5. Tsuyuoka R, Bailey JW, Guimarães AM, Gurgel RQ, Cuevas LE. Anemia and intestinal parasitic infections in primary school students in Aracaju, Sergipe, Brazil. Cad Saude Publica. 1999;15:413-21.

6. Stephenson LS, Latham MC, Ottesen EA. Malnutrition and parasitic helminth infections. Parasitol. 2000;121:23-38.

7. Thomas M, Woodfield G, Moses C, Amos G. Soil-transmitted helminth infection, skin infection, anaemia, and growth retardation in schoolchildren of Taveuni Island, Fiji. $\mathrm{N} Z$ Med J. 2005;118:U1492.

8. Bethony J, Brooker S, Albonico M, Geiger SM, Loukas A, Diemert D, et al. Soil transmitted infections: ascariosis, trichuriasis and hookworm. Lancet. 2006;367:1521-32.

9. Costamagna SR, García S, Visciarelli E, Casas N. Epidemiología de las parasitosis en Bahía Blanca (Provincia de Buenos Aires) Argentina, 1994/1999. Parasitol Latinoam. 2002;57: 103-10.

10. Lura MC, Beltramino DM, De Carrera EF. Prevalence of intestinal helminthiasis in primary school children in Santa Fe city. Medicina (Buenos Aires). 2002;62:29-36.

11. Gamboa MI, Basualdo JA, Córdoba MA, Pezzani BC, Minvielle MC, Lahitte HB. Distribu- tion of intestinal parasitoses in relation to environmental and sociocultural parameters in La Plata, Argentina. J Helminthol. 2003;77: 15-20.

12. Taranto NJ, Cajal SP, De Marzi MC, Fernández M, Frank FM, Bru AM, et al. Clinical status and parasitic infection in a Wichi Aboriginal community in Salta, Argentina. Trans R Soc Trop Med Hyg. 2003;97:554-8.

13. Navone GT, Gamboa MI, Oyhenart EE, Orden AB. Parasitosis intestinales en poblaciones Mbya-Guaraní de la provincia de Misiones. Aspectos epidemiológicos y nutricionales. Cad Saude Publica. 2006;22:109-18.

14. Alonso JM, Stein M, Chamorro MC, Bojanich MV. Contamination of soils with eggs of Toxocara in a subtropical city in Argentina. J Helminthol. 2001;75:165-8.

15. Córdoba A, Ciarmela L, Pezzani B, Gamboa M, De Luca M, Minviele M, et al. Presencia de parásitos intestinales en paseos públicos urbanos en La Plata, Argentina. Parasitol Latinoam. 2002;57:25-9.

16. Lechner L, Amalfitano G, Madrid V, Denegri G, Sardella N. Relevamiento parasitológico en areneros de plazas y paseos públicos de la ciudad de Mar del Plata. Acta Bioquím Clín Latinoam. 2003;1:85.

17. Pierangeli NB, Giayetto AL, Manacorda AM, Barbieri LM, Soriano SV, Veronesi A, et al. Estacionalidad de parásitos intestinales en suelos periurbanos de la ciudad de Neuquén, Patagonia, Argentina. Trop Med Int Health. 2003;8:259-63.

18. Zunino MG, De Francesco MV, Kuruc JA, Schweigmann N, Wisnivesky-Colli MC, Jensen $\mathrm{O}$. Contamination by helminths in public places of the province of Chubut, Argentina. Bol Chil Parasitol. 2000;55:78-83.

19. Minvielle M, Taus MR, Ciarmela ML, Francisconi M, Barlasina M, Pezzani BC, et al. Aspectos epidemiológicos asociados a toxocarosis en Gualeguaychú, Entre Ríos, Argentina. Parasitol Latinoam. 2003;58:128-30.

20. Rubel D, Wisnivesky C. Magnitude and distribution of canine fecal contamination and helminth eggs in two areas of different urban structure, Greater Buenos Aires, Argentina. Vet Parasitol. 2005;133:339-47.

21. Sánchez P, Raso S, Torrecillas C, Mellado I, Ñancufil A, Oyarzo CM, et al. Contaminación biológica con heces caninas y parásitos intestinales en espacios públicos urbanos de dos ciudades de la Provincia de Chubut, Patagonia Argentina. Parasitol Latinoam. 2003;58:131-5.

22. Milano A, Oscherov E. Contaminación de aceras con enteroparásitos caninos en Corrientes, Argentina. Parasitol Latinoam. 2005;60:82-5.

23. Gamboa MI. Effects of temperature and humidity on the development of the eggs of Toxocara canis in laboratory conditions. J Helminthol. 2005;79:327-31.

24. Hurtado MA, Giménez JE, Cabral MA. Análisis ambiental del partido de La Plata: aportes al ordenamiento territorial. Buenos Aires: Consejo Federal de Inversiones; 2006.

25. Gamboa MI, Basualdo Farjat JA, Kozubsky L, Costas ME, Cueto Rúa E, Lahitte HB. Prevalence of intestinal parasitosis within three population groups in La Plata, Buenos Aires, Argentina. Eur J Epidemiol. 1998;14:55-61.

26. World Health Organization. Basic laboratory methods in medical parasitology. Geneva: WHO; 1991.

27. Navone GT, Gamboa MI, Kozubsky LE, Costas ME, Cardozo MI, Sisliauskas MN, et al. Estudio comparativo de recuperación de formas parasitarias por diferentes métodos de enriquecimiento coproparasitológico. Parasitol Latinoam. 2005;60:178-81.

28. Centers for Disease Control and Prevention. Epi Info version 3.3.2. Atlanta, GA: CDC; 2005. Hallado en http://www.cdc.gov/ epiinfo/. Acceso el 16 de junio de 2009.

29. Martínez MR, Pochettino ML, Arenas PM. La horticultura: estrategia de subsistencia en contextos pluriculturales, Valle del Cuñapirú, Misiones, Argentina. Delpinoa. 2003;45:89-97.

30. Lawrence DN, Neel JV, Abadie SH, Moore LL, Adams LJ, Healy GR, et al. Estudios epidemiológicos entre populações amerindias da 
Amazônia. Parasitoses intestinais in povoações recentemente contactadas e em aculturação. Acta Amazon. 1983;13:393-407.

31. Miranda RA, Xavier FB, Menezes RC. Parasitismo intestinal e desnutrição proteicoenergética em crianças menores de 10 anos de idade de duas aldeias indígenas na tribo Parakaná, sudeste do Estado do Pará, Brasil. Arq Bras Ped. 1997;4:169-73.

32. Miranda RA, Xavier FB, Nascimento JR, Menezes RC. Frecuencia de parasitismo intestinal nas aldeias indígenas da tribo Tembe, Amazonia Oriental Brasileira. Rev Soc Bras Med Trop. 1999;32:389-93.

33. Soriano SV, Barbieri LM, Pierángeli NB, Giayetto $\mathrm{AL}$, Manacorda $\mathrm{AM}$, Castronovo E, et al. Intestinal parasites and the environment: frequency of intestinal parasites in children of Neuquén, Patagonia, Argentina. Rev Latinoam Microbiol. 2001;43:96-101.

34. Borda CE, Rea MJ, Rosa JR, Maidana C. Intestinal parasitism in San Cayetano, Corrientes, Argentina. Bull Pan Am Health Organ. 1996;30:227-33.

35. Sorensen E, Marhoof I, Amarasinghe D, Hettiarachchi I, Dassenaieke TS, et al. The effect of availability of latrines on soil-transmitted nematode infections in the plantation sector in Sri Lanka. Am J Trop Med Hyg. 1994;1: 36-9.

36. Ashford RW, Craig PS, Oppenheimer SJ. Polyparasitism on the Kenya coast. 1. Prevalence and association between parasitic infections. Ann Trop Med Parasitol. 1992;86:671-9.

37. Minvielle MC, Pezzani, BC, Basualdo JA. Frequency of finding helminth eggs in canine stool samples collected in public places from La Plata city, Argentina. Bol Chil Parasitol. 1993; 48:63-7.

38. Basualdo J, Córdoba MA, De Luca MM, Pezzani B, Ciarmela ML, Grenovero MS, et al. Intestinal parasitoses and environmental factors in al rural population of Argentina, 2002-2003. Rev Inst Med Trop Sao Paulo. 2007;49:251-5.

Manuscrito recibido el 10 de enero de 2008. Aceptado para publicación, tras revisión, el 22 de octubre de 2008.

ABSTRACT Objectives. To analyze the relationship between the geohelminth species found in urban, suburban, and rural areas of the Buenos Aires and Misiones provinces of Argentina, and the socioenvironmental conditions that promote infection by these parasites.

Associations between geohelminths and socioenvironmental conditions among different human populations in Argentina

Methods. This was a cross-sectional study that analyzed 700 human fecal samples taken from 319 families residing in an urban population (UP) and two suburban ones (SUP1, SUP2) in the Province of Buenos Aires, and a rural one in Misiones (RP). Thirty-five samples of dog feces and 205 earth samples were taken, plus surveys were performed of the socioenvironmental characteristics of the study areas. The Ritchie, Carles-Barthelemy, Fülleborn, and Kato-Katz parasite analysis techniques were used. Results. The highest incidence of parasites was in RP $(78.4 \%)$, followed by the suburban areas SUP1 (35.0\%) and SUP2 (25.8\%), and lastly, the urban area (5.7\%). Ancylostomatidae $(71.1 \%)$ and Strongyloides stercoralis $(22.2 \%)$ were found only in RP, while Ascaris lumbricoides, Hymenolepis nana, and Trichuris trichiura were most frequent in SUP1. The samples of dog feces from the Misiones province had the highest incidence of parasites (100\%) compared to those from Buenos Aires, but the urban dirt from this city proved to be more contaminated. Poor hygiene habits in food preparation and toileting, overcrowding, walking barefoot, and dwellings with dirt floors were significantly associated with a higher frequency of intestinal geohelminths and pseudogeohelminths $(P<0.05)$.

Conclusions. The great number of cases of multiple infestations seen among suburban as well as rural populations calls for special attention and underscores the need for more extensive epidemiological studies that take on the different aspects of this complex issue with the goal of implementing more efficient health initiatives and programs. 